\title{
Inhibitory Effect of S100A8 on Neutrophil Apoptosis by Cytokine Release of Normal and Allergic Monocytes
}

\author{
Ji-Sook Lee ${ }^{\dagger ; *}$ \\ Department of Clinical Laboratory Science, Wonkwang Health Science University, Iksan 54538, Korea
}

\begin{abstract}
S100A8 functions as an essential factor in inflammatory response. Cytokine release of monocytes and regulation of neutrophil apoptosis are important steps in pathogenesis of allergy. This study aims to examine the relation between cytokine release of monocytes due to S100A8 and neutrophil apoptosis. S100A8 enhanced the release of IL-6 and IL-8 in monocytes of normal and allergic subjects. Treatment of supernatants of normal and allergic monocytes with S100A8 blocked neutrophil apoptosis by inhibition of caspase 9 and caspase 3 activation. The secretion signal induced by S100A 8

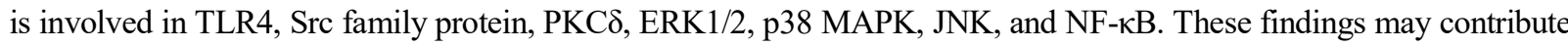
to understanding the complex pathogenesis of allergic diseases by determining inflammatory responses associated with S100A8, monocytes, and neutrophils.
\end{abstract}

Key Words: Allergy, Monocytes, Neutrophils apoptosis, S100

S100A8, known as MRP8, is included in the S100 protein family and expressed in various cell types, including neutrophils, monocytes, and myeloid dendritic cells (Goyette and Geczy, 2011). Intracellular S100A8 functions as a scavenger of reactive oxygen species (ROS) and telomerase activity after intracellular calcium binding. Extracellular S100A8 regulates $\beta 2$ integrin-dependent neutrophil chemotaxis and expression of IL-1 $\beta$ and TNF- $\alpha$ in Toll-like receptor (TLR4)dependency (Donato et al., 2013). In monocytes, S100A8 induces cell migration by microtubule reassembly (Vogl et al., 2004). A recent study reported on the activation of monocytes due to S100A8 in TLR4-dependency using transcriptome evaluation (Fassl et al., 2015). Pathogenesis of allergic diseases is elicited by a variety of causes including gene mutation, toxic environment, and immune dysregulation (Holgate, 2008). Cytokine secretion and inhibition of neutro- phil apoptosis increase inflammatory responses, resulting in exacerbation of allergy, and association of S100A8 and allergic diseases such as asthma and atopic dermatitis has been reported (Kim et al., 2014). We have, for the first time, demonstrated that S100A8 secreted by house dust mite directly inhibits neutrophil apoptosis in normal and asthmatic subjects (Kim et al., 2013).

A total of 10 allergy patients including 5 allergic asthma and 5 allergic rhinitis subjects (average $=31.6$ years) were recruited from Eulji University Hospital. Allergic status was based on the presence of positive results of a skin prick test $(\geq 2+)$, multiple allergen simultaneous test (MAST) $(\geq$ clas2), or measurement of specific HDM IgE using the Pharmacia Unicap 100 system to common allergens. Levels of total IgE of normal and allergic subjects using an ADVIA Centaur immunoassay (Siemens Medical Solutions Diag-

Received: July 24, 2020 / Revised: September 7, 2020 / Accepted: September 7, 2020

* Professor.

${ }^{\dagger}$ Corresponding author: Ji-Sook Lee. Department of Clinical Laboratory Science, Wonkwang Health Science University, 501, Iksandaero, Iksan 54538, Korea.

Tel: +82-63-840-1216, Fax: +82-63-840-1219, e-mail: jslee1216@wu.ac.kr

(C) The Korean Society for Biomedical Laboratory Sciences. All rights reserved.

(c) This is an Open Access article distributed under the terms of the Creative Commons Attribution Non-Commercial License (http://creativecommons.org/licenses/by-nc/3.0/) which permits unrestricted non-commercial use, distribution, and reproduction in any medium, provided the original work is properly cited. 
(A)

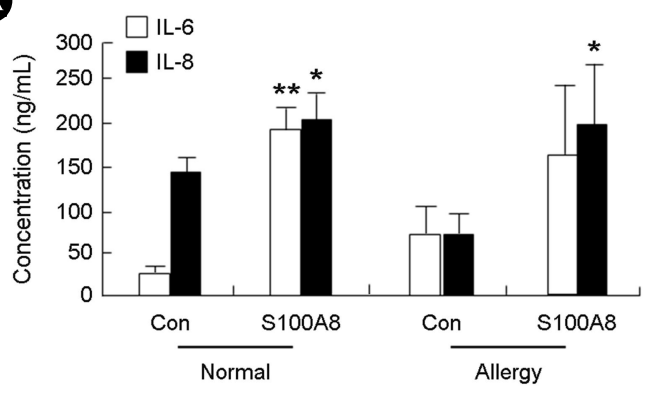

B

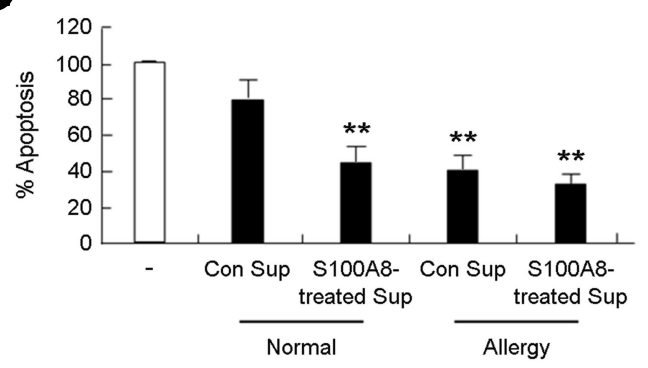

C
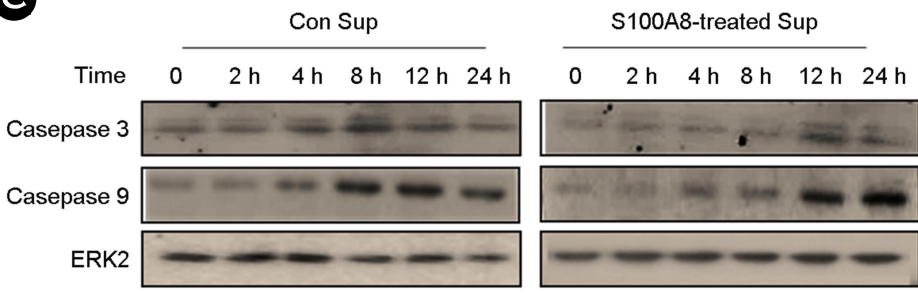

Fig. 1. S100A8 induces IL-6, and IL-8 and cytokine secretion suppresses neutrophil apoptosis via inhibition of the caspase 9/3 pathway. (A) Monocytes isolated from normal and allergic subjects were incubated in the absence or presence of $1 \mu \mathrm{g} / \mathrm{mL} \mathrm{S100A8} \mathrm{for} \mathrm{the} \mathrm{indicated} \mathrm{time.} \mathrm{(B)}$ Monocytes were incubated with and without $1 \mu \mathrm{g} / \mathrm{mL}$ of S100A8 for $48 \mathrm{~h}$. The supernatant (Sup) was collected and added to the fresh neutrophils isolated from normal and allergic subjects. Neutrophils apoptosis was analyzed by measuring the binding of annexin V-FITC and PI. Data are presented relative to the control, which was set at $100 \%$ as the means \pm S.E.M. ${ }^{* *} P<0.01$ indicates a significant difference between the control and S100A8-treated groups or between the control supernatant and supernatanttreated groups. (C) Normal neutrophils were incubated with the supernatant (Sup) or the S100A8-treated supernatant of normal monocytes. Caspase 9 and caspase 3 were detected by Western blotting. ERK2 expression was used as an internal control. nostics, Erfurt, Germany) were $68.5 \mathrm{IU} / \mathrm{mL}$ and $565.3 \mathrm{IU} / \mathrm{mL}$, respectively. Additionally, 8 normal subjects between 18 and 30 years of age (average $=23.0$ years) were recruited as controls. This study was approved by the Institutional Review Board of Eulji University for normal volunteers allergic subjects (EU14-33). All participants in this study gave their written informed consent. Human monocytes and neutrophils were isolated from the heparinized peripheral blood of healthy persons and allergic subjects using Ficoll-Hypaque gradient centrifugation (Kim et al., 2014). Both a CD16 microbeads magnetic cell sorting kit and monocyte isolation kit II (Miltenyi Biotec, Bergisch Gladbach, Germany) were used for isolation of neutrophil and monocyte, respectively. The cells were washed after hypotonic lysis to remove erythrocytes. This method routinely yielded greater than 97\% purity of neutrophil and monocyte, respectively. An annexin V-fluorescein isothiocyanate (FITC) apoptosis detection kit (BD Biosciences, San Diego, CA, USA) was used for detection of neutrophil apoptosis. Activation of caspase 9 and 3, and were confirmed by Western blotting. The concentrations of IL-6 and IL-8 in a cell supernatant after treatment with S100A8 for $48 \mathrm{~h}$ were measured with a sandwich enzymelinked immunosorbent assay (ELISA) using OptEIA ${ }^{\mathrm{TM}}$ Set human IL-6 and IL-8 (BD Biosciences, San Diego, CA, USA). The DNA-binding activity of NF- $\mathrm{BB}$ was evaluated using EZ-Detect ${ }^{\mathrm{TM}}$ transcription factor kits for NF- $\mathrm{kB}$ p65 (PIERCE, Rockford, IL) as described in the manufacturer's instructions. DNA binding specificity was assessed using wild type or mutant NF-KB oligonucleotides. Chemiluminescent detection was performed using a luminometer. Here, we hypothesized that S100A8 induces the secretion of cytokines associated with neutrophil survival such as IL-6 and IL-8 in monocytes, and the cytokine release regulates constitutive apoptosis of neutrophils. To determine whether or not the effect of S100A8 on cytokine release occurs in allergic diseases, we evaluated the change of secretion of IL-6 and IL-8 due to S100A8 in monocytes separated from normal and allergic subjects. S100A8 induced the elevation of IL-6 and IL-8 in normal and allergic monocytes compared with control (Fig. 1A). Supernatant treated with S100A8 inhibited apoptosis of neutrophils isolated from normal peripheral blood, while control supernatant without S100A8 treatment did not affect neutrophil apoptosis (Fig. 1B). Supernatant treated with S100A8 suppressed allergic neutrophil apoptosis. Control supernatant of allergic monocytes without S100A8 treatment also inhibited the apoptosis of allergic 
neutrophils in contrast to the normal result. Activation of caspase 9 and caspase 3 was inhibited by S100A8 in a timedependent manner (Fig. 1C). TLR4i, an inhibitor of TLR4, PP2, an inhibitor of Src family protein, rottlerin, an inhibitor of PKC $\delta$, PD98059, an inhibitor of ERK, SB202190, an inhibitor of p38 MAPK, SP600125, an inhibitor of JNK and BAY-11-7085, an inhibitor of NF- $\mathrm{B}$ significantly blocked the increased expression of IL-6 and IL-8 $(P<0.05)$ (Fig. 2A). Activation of NF- $\mathrm{kB}$ induced by S100A8 was significantly inhibited by TLR4i, PP2, rottlerin, PD98059, SB202190, and SP600125 $(P<0.05)$ (Fig. 2B). S100A8 functions as a pathological factor in induction of allergic diseases, autoimmune diseases, and malignant tumors (Jin et al., 2014; Kang et al., 2015). However, a few research groups have demonstrated that S100A8 plays a role as a protector in pathogenesis of allergy. S100A8 reduces mast cell degranulation and eosinophil chemotaxis in ovalbumin-sensitized mice, and the S100A8 concentration of induced sputum in

A
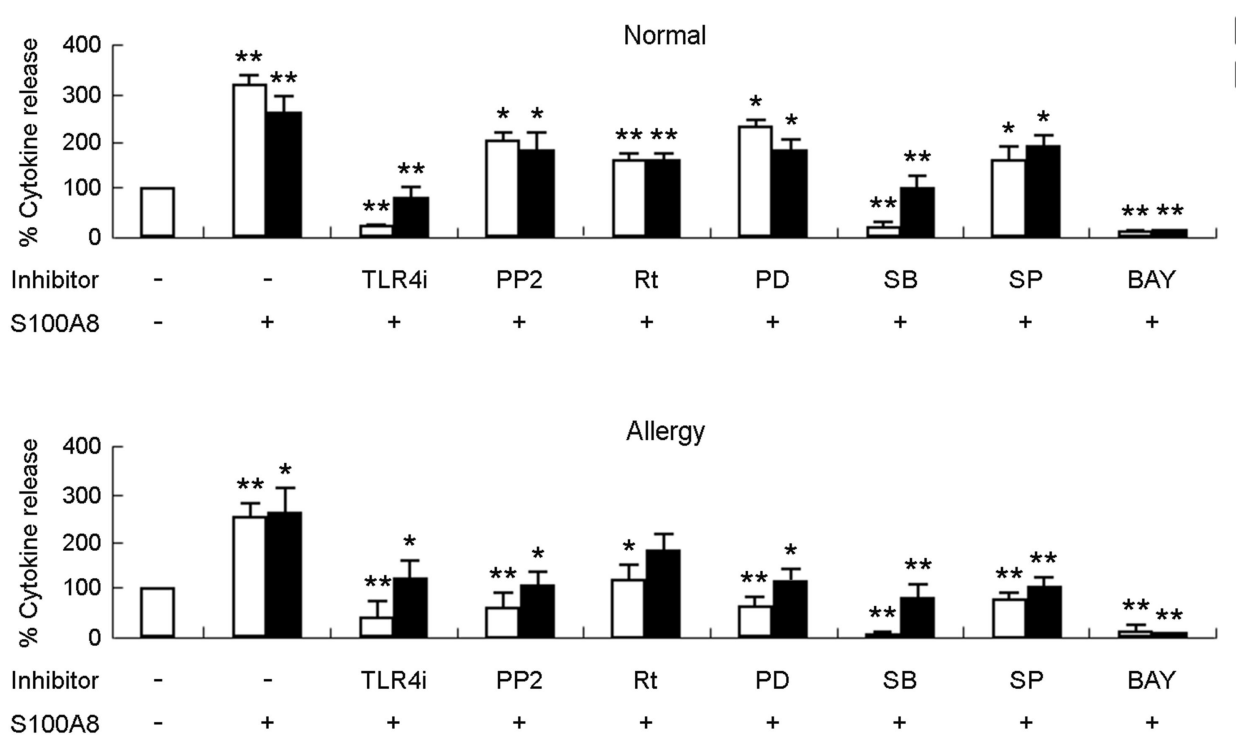

B

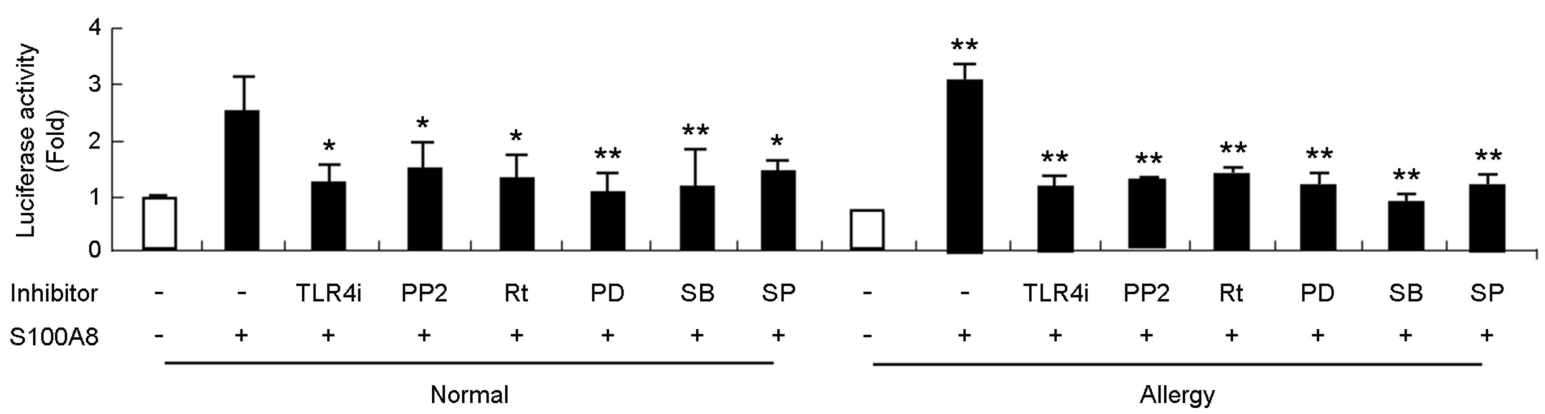

Fig. 2. Cytokine secretion enhanced by S100A8 is involved in TLR4, Src family protein, PKCס, MAPKs, and NF-אB in normal and allergic monocytes. (A) Normal (upper panel) and allergic monocytes (lower panel) were pre-treated for $1 \mathrm{~h}$ with and without $2 \mu \mathrm{M}$ TLR4i, $20 \mu \mathrm{M}$ PP2, $5 \mu \mathrm{M}$ rottlerin, $20 \mu \mathrm{M}$ PD98059 (PD), $20 \mu \mathrm{M}$ SB202190 (SB), $20 \mu \mathrm{M}$ SP600125 (SP) and $10 \mu \mathrm{M}$ BAY-11-7085 (BAY), after which the cells were incubated for $48 \mathrm{~h}$ in the absence and presence of S100A8 $(1 \mu \mathrm{g} / \mathrm{mL})$. The supernatant was collected and analyzed by ELISA. (B) Normal monocytes were pre-treated for $1 \mathrm{~h}$ with and without $2 \mu \mathrm{M}$ TLR4i, $20 \mu \mathrm{M}$ PP2, $5 \mu \mathrm{M}$ rottlerin, $20 \mu \mathrm{M}$ PD98059 (PD), $20 \mu \mathrm{M}$ SB202190 (SB) and $20 \mu \mathrm{M}$ SP600125 (SP), after which the cells were incubated with S100A8 (1 $\mu \mathrm{g} / \mathrm{mL})$ for $1 \mathrm{~h}$. After harvested cells were lysed, NF- $\mathrm{kB}$ in the lysates was detected by luciferase assay. Data are expressed as the means \pm S.E.M. $* P<0.05$ and $* * P<0.01$ indicate a significant difference between the control and S100A8-treated groups or between the S100A8-treated and inhibitor-treated groups. 
asthma patients was lower than in normal subjects (Zhao et al., 2011). Our results showed that S100A8 induced the expression of IL-6 and IL-8 in normal and allergic monocytes (Fig. 1A). Cytokines secreted by S100A8 have an antiapoptotic effect on neutrophil apoptosis, which is mediated by inhibition of caspase 9 and caspase 3 (Figs. 2A and B). IL-6 and IL-8 are neutrophil survival factors and pleiotropic cytokines, which affect transition of allergic phase, activation of stromal cells, and chemotaxis (Kim and Lee, 2017). Our study supports that S100A8 promotes a pro-inflammatory effect including cytokine release and inhibition of neutrophil apoptosis in allergic diseases. These results match with our previous data related to S100A9, which binds to TLR4 (Lee et al., 2016). Apoptosis of allergic neutrophils is inhibited by control supernatant, but not that of normal neutrophils. These results indicate that response of neutrophils to factors secreted from monocytes differs between normal and allergic monocytes. Because the number of normal and allergic subjects is limited in this study, we will proceed in our study with an increased number of experimental subjects and also examine the effects of S100A8 depending on allergenspecific subjects such as existence of total $\operatorname{IgE}$ and house dust mite-specific IgE.

\section{ACKNOWLEDGEMENT}

This paper was supported by Wonkwang Health Science University in 2020.

\section{CONFLICT OF INTEREST}

The authors have no conflicts of interest, financial or otherwise, to declare.

\section{REFERENCES}

Donato R, Cannon BR, Sorci G, Riuzzi F, Hsu K, Weber DJ, Geczy CL. Functions of S100 proteins. Current Molecular Medicine. 2013. 13: 24-57.

Fassl SK, Austermann J, Papantonopoulou O, Riemenschneider M, Xue J, Bertheloot D, Freise N, Spiekermann C, Witten A, Viemann D, Kirschnek S, Stoll M, Latz E, Schultze JL, Roth J, Vogl T. Transcriptome assessment reveals a dominant role for TLR4 in the activation of human monocytes by the alarmin MRP8. Journal of Immunology. 2015. 194: 575-583.
Goyette J, Geczy CL. Inflammation-associated S100 proteins: new mechanisms that regulate function. Amino Acids. 2011. 41: 821-842.

Holgate ST. Pathogenesis of asthma. Clinicanl and Experimental Allergy 2008. 38: 872-897.

Jin S, Park CO, Shin JU, Noh JY, Lee YS, Lee NR, Kim HR, Noh S, Lee Y, Lee JH, Lee KH. DAMP molecules S100A9 and S100A8 activated by IL-17A and house-dust mites are increased in atopic dermatitis. Experimental Dermatology. 2014. 23: 938-941.

Kang JH, Hwang SM, Chung IY. S100A8, S100A9 and S100A12 activate airway epithelial cells to produce MUC5AC via extracellular signal-regulated kinase and nuclear factor- $\kappa \mathrm{B}$ pathways. Immunology. 2015. 144: 79-90.

Kim EH, Lee JS, Lee NR, Baek SY, Kim EJ, Lee SJ, Kim IS. Regulation of Constitutive Neutrophil Apoptosis Due to House Dust Mite Allergen in Normal and Allergic Rhinitis Subjects. PLoS One. 2014. 9: e105814.

Kim IS, Kim MJ, Kim DH, Choi E, Lee JS. Different anti-apoptotic effects of normal and asthmatic serum on normal eosinophil apoptosis depending on house dust mite-specific IgE. Molecular Biology Reports. 2013. 40: 5875-5881.

Kim IS, Lee JS. S100A8 and S100A9 secreted by allergens in monocytes inhibit spontaneous apoptosis of normal and asthmatic neutrophils via the Lyn/akt/ERK pathway. Korean Journal of Clinical Laboratory Science. 2017. 49: 128-134.

Lee NR, Park BS, Kim SY, Gu A, Kim DH, Lee JS, Kim IS Cytokine secreted by S100A9 via TLR4 in monocytes delays neutrophil apoptosis by inhibition of caspase 9/3 pathway. Cytokine. 2016. 86: 53-63.

Vogl T, Ludwig S, Goebeler M, Strey A, Thorey IS, Reichelt R, Foell D, Gerke V, Manitz MP, Nacken W, Werner S, Sorg C, Roth J. MRP8 and MRP14 control microtubule reorganization during transendothelial migration of phagocytes. Blood. 2004 104: 4260-4268

Zhao J, Endoh I, Hsu K, Tedla N, Endoh Y, Geczy CL. S100A8 modulates mast cell function and suppresses eosinophil migration in acute asthma. Antioxidant Redox Signal. 2011. 14: 1589 -1600 .

https://doi.org/10.15616/BSL.2020.26.3.226

Cite this article as: Lee JS. Inhibitory Effect of S100A8 on Neutrophil Apoptosis by Cytokine Release of Normal and Allergic Monocytes. Biomedical Science Letters. 2020. 26: 226-229. 